Check for updates

Cite this: RSC Adv., 2018, 8, 7550

Received 10th January 2018

Accepted 12th February 2018

DOI: $10.1039 / \mathrm{c} 8 \mathrm{ra00257f}$

rsc.li/rsc-advances

\section{Luminescence and photoelectrochemical properties of size-selected aqueous copper-doped Ag-In-S quantum dots $\uparrow$}

\author{
Alexandra Raevskaya, ${ }^{\text {ab }}$ Oksana Rozovik, ${ }^{a}$ Anastasiya Novikova, ${ }^{c}$ \\ Oleksandr Selyshchev, ${ }^{d}$ Oleksandr Stroyuk, (D) *ab Volodymyr Dzhagan, (D) e \\ Irina Goryacheva, ${ }^{c}$ Nikolai Gaponik, (D) ${ }^{\mathrm{b}}$ Dietrich R. T. Zahn ${ }^{\mathrm{d}}$ \\ and Alexander Eychmüller (iD ${ }^{\mathrm{b}}$
}

Ternary luminescent copper and silver indium sulfide quantum dots (QDs) can be an attractive alternative to cadmium and lead chalcogenide QDs. The optical properties of $\mathrm{Cu}-\mathrm{ln}-\mathrm{S}$ and $\mathrm{Ag}-\mathrm{ln}-\mathrm{S}$ (AIS) QDs vary over a broad range depending on the QD composition and size. The implementation of ternary QDs as emitters in bio-sensing applications can be boosted by the development of mild and reproducible syntheses directly in aqueous solutions as well as the methods of shifting the photoluminescence (PL) bands of such QDs as far as possible into the near IR spectral range. In the present work, the copper-doping of aqueous nonstoichiometric AIS QDs was found to result in a red shift of the PL band maximum from around $630 \mathrm{~nm}$ to $\sim 780 \mathrm{~nm}$ and $\mathrm{PL}$ quenching. The deposition of a ZnS shell results in PL intensity recovery with the highest quantum yield of $15 \%$, with almost not change in the PL band position, opposite to the undoped AIS QDs. Size-selective precipitation using 2-propanol as a non-solvent allows discrimination of up to 9 fractions of Cu-doped AIS/ZnS QDs with the average sizes in the fractions varying from around 3 to $2 \mathrm{~nm}$ and smaller and with reasonably the same composition irrespective of the QD size. The decrease of the average QD size results in a blue PL shift yielding a series of bright luminophors with the emission color varies from deep-red to bluish-green and the PL efficiency increases from $11 \%$ for the first fraction to up to $58 \%$ for the smallest Cu-doped AIS/ZnS QDs. The rate constant of the radiative recombination of the size-selected Cu-doped AIS/ZnS QDs revealed a steady growth with the QD size decrease as a result of the size-dependent enhancement of the spatial exciton confinement. The copper doping was found to result in an enhancement of the photoelectrochemical activity of CAIS/ZnS QDs introduced as spectral sensitizers of mesoporous titania photoanodes of liquid-junction solar cells.

\section{Introduction}

Metal chalcogenide nanocrystals with a size smaller than the doubled Bohr exciton radius, or quantum dots (QDs), reveal in many cases a unique combination of intense light absorbance, photoluminescence (PL) emission with high quantum yields (QYs), and a strong dependence of the bandgap $\left(E_{\mathrm{g}}\right)$ and the energies of the charge carriers on the QD size. This allows for

${ }^{a}$ L. V. Pysarzhevsky Institute of Physical Chemistry, National Academy of Sciences of Ukraine, Kyiv, 03028, Ukraine.E-mail: alstroyuk@ukr.net

${ }^{b}$ Physical Chemistry, TU Dresden, 01062 Dresden, Germany. E-mail: oleksandr. stroyuk@chemie.tu-dresden.de

${ }^{c}$ Saratov State University, 410012, Saratov, Russian Federation

${ }^{d}$ Semiconductor Physics, Chemnitz University of Technology, 09107 Chemnitz, Germany

${ }^{e}$ V. E. Lashkaryov Institute of Semiconductors Physics, National Academy of Sciences of Ukraine, Kyiv, 03028, Ukraine

† Electronic supplementary information (ESI) available. See DOI: $10.1039 / \mathrm{c} 8 \mathrm{ra00257f}$ a broad variation of spectral, photophysical, and photochemical properties of such QDs by tailoring their size at a constant chemical composition. These properties of the metalchalcogenide QDs might be introduced into a variety of lightharvesting and light-emitting applications, in particular, into photovoltaics, luminescent bio-labeling, light-emitting diodes, light detectors and concentrators, etc. $^{1-5}$ Typically, the research on metal-chalcogenide QDs is focused on cadmium $(\mathrm{CdX}, \mathrm{X}=\mathrm{S}$, $\mathrm{Se}, \mathrm{Te}$ ) and lead chalcogenides ( $\mathrm{PbX}$ ) capable of intense light absorption in the visible (CdX) and near-infrared $(\mathrm{PbX})$ ranges and intensely emitting with narrow PL bands with PL QYs as high as $90-95 \%{ }^{4-6}$

However, the toxicity of $\mathrm{CdX} / \mathrm{PbX}$ QDs and the products of their corrosion stimulates a search for alternative compounds with similar spectral characteristics and strong sizedependences of the electronic properties, in particular, among ternary and more complex chalcogenides of In, $\mathrm{Ga}, \mathrm{Sn}$, etc. $^{4,7-16}$ This search focused on ternary sulfides $\mathrm{CuInS}_{2}$ (CIS) and AgInS (AIS) having narrow bandgaps of $1.5 \mathrm{eV}$ and $1.85 \mathrm{eV}$, 
respectively ${ }^{\mathbf{7 - 9 , 1 3 , 1 5}}$ and being well suited for solar light harvesting. Also, CIS and AIS QDs can emit strong PL with the spectral parameters broadly varying with the CIS/AIS QD composition, size, doping, etc. ${ }^{7,8,11-15}$ Such ternary compounds revealed a number of quite unique properties differing drastically from those of the binary $\mathrm{CdX} / \mathrm{PbX}$ compounds, in particular, the immensely broad deviations from the stoichiometry while preserving the crystal lattice symmetry and quality, the capability to form a plethora of solid solutions via a partial substitution of sulfur with Se or Te, In - with Ga, and by alloying CIS (AIS) with ZnS, as well as the lattice preservation at a heavy doping with "alien" metal cations., ${ }^{7,9-16}$ These special features open many options for tailoring the band structure of the ternary compounds with related consequences as to the spectral sensitivity range, the charge carriers energies, the conductivity and the carrier mobility, etc., which are unattainable for the binary compounds. This unprecedented variability of properties becomes even broader in the nanometer crystal size range, where size dependences of the electronic properties become expressed distinctly. ${ }^{9-16}$

Typically, the CIS (AIS) QDs are produced by “classic” heating $\mathrm{up} /$ hot injection approaches, developed in detail for $\mathrm{CdX} / \mathrm{PbX}$ QDs, when the nucleation and ripening of the QDs occur in the high-boiling solvents playing simultaneously the role of capping ligands (such as trioctylphosphine oxide or oleylamine) or/and as sulfur source (1-dodecanethiol). ${ }^{\mathbf{1 , 4 , 5 1 7 - 2 1}}$ These methods allow for a precise control over the QD size, size distribution and phase composition. However, many applications favor (like e.g. photovoltaics) or even demand (e.g. luminescent bio-labeling) the post-synthesis transfer of CIS (AIS) QDs into water. Conventionally, the transfer is performed by introducing bifunctional molecules, such as mercaptoacids, simultaneously being capable to bind strongly to the QD surface via the mercapto-group and to stabilize the QDs in water via the deprotonation of the carboxyl group providing an electrostatic shield against inter-particle interactions. ${ }^{3,4,7,9,11-16,22}$

To avoid such a two-step synthetic procedure and make the total synthesis more environmentally friendly the approaches to the synthesis of CIS (AIS) QDs directly in aqueous solutions have received recently an ever-growing attention.,3,10,23-26 The research in this area has already yielded synthetic protocols for the preparation of strongly absorbing QD "inks" for the utilization as light-harvesting components of solar cells. However, the PL QYs of CIS (AIS) QDs produced directly in water still remain low and a further exploration is needed to improve the control over the QD size, surface chemistry and lattice perfection in aqueous syntheses of ternary QDs.

Recently we have reported on the aqueous synthesis and size selection of brightly luminescent AIS and core/shell AIS/ZnS QDs stabilized by mercaptoacetate (MA) anions. ${ }^{27}$ The sizeselected AIS/ZnS QDs emit light with relatively high QYs of $45-46 \%$ for the best fractions, however, the spectral PL range is limited to around $700 \mathrm{~nm}$ (in terms of the PL band maximum position).

At the same time, many applications, such as light-emitting devices and luminescent bio-sensing in the first transparency window of the human skin require luminophors emitting in the near IR range. To extend the spectral range of the emission doping with $\mathrm{Cu}$ or alloying between AIS and CIS is proposed frequently. ${ }^{\mathbf{1 4 , 1 5 , 2 8}} \mathrm{Cu}$ doping was also found to be an efficient method of improving the charge carrier mobility in metalchalcogenide QDs, in particular in AIS QDs, making them more efficient light harvesters in third-generation solar cells. ${ }^{29-31}$ Incorporation of copper ions into alloyed $\mathrm{ZnS}^{-} \mathrm{In}_{2} \mathrm{~S}_{3}$ (ref. 32 and 33) and $\mathrm{Zn}-\mathrm{In}-\mathrm{Se}^{34}$ nanocrystals was found to result in a spectacular enhancement of the photocatalytic properties and PL efficiency, respectively. $\mathrm{Cu}$ doping of AIS and AIS/ZnS QDs synthesized by the heating-up method results in a shift of the emission to lower energies and was used to produce efficient luminescent markers for the imaging of tumor cells. ${ }^{23,35}$

In the present paper we summarize our studies on Cu-doped AIS (CAIS) and CAIS/ZnS QDs synthesized in aqueous solutions and subjected to post-synthetic size selection. Our results indicate that the Cu doping is an attractive method for tailoring the PL range allowing to shift the PL band down to $800 \mathrm{~nm}$ and still have a reasonably high PL QY. The size selection was found to be a potent tool of influencing both the spectral PL parameters and the PL efficiency, yielding CAIS/ZnS QDs with PL QYs reaching almost $60 \%$.

\section{Materials and methods}

\section{Chemicals}

Indium(III) chloride, silver and copper(II) nitrates, zinc(II) acetate dihydrate, $\mathrm{Na}_{2} \mathrm{~S} \cdot 9 \mathrm{H}_{2} \mathrm{O}, \mathrm{NH}_{4} \mathrm{OH}$ (aqueous $5.0 \mathrm{M}$ solution), mercaptoacetic acid (MAA), 2-propanol were purchased from SigmaAldrich and Acros Organics and used without additional purification. All the solutions were prepared using deionized (DI) Milli-Q water (Millipore).

\section{Synthesis and size-selective precipitation of QDs}

Aqueous colloidal solutions of AIS and CAIS QDs were prepared by a reaction between sodium sulfide and a mixture of silver(I) or silver(I) + copper(II) and indium(III) MA complexes similarly to our other reports. ${ }^{27,36}$ In a typical synthesis, the feed molar ratio of silver to indium to sulfur, $\mathrm{Ag}: \mathrm{In}: \mathrm{S}$, was adjusted to $2: 7: 10$. $2.0 \mathrm{~mL}$ aqueous $0.1 \mathrm{M} \mathrm{AgNO}_{3}$ solution, an aliquot (for example, $0.2 \mathrm{~mL}$ ) of aqueous $0.1 \mathrm{M} \mathrm{Cu}\left(\mathrm{NO}_{3}\right)_{2}$ solution, and $2 \mathrm{~mL}$ aqueous 1.0 M MAA solution were added to $94 \mathrm{~mL}$ water under magnetic stirring and ambient conditions. The resulting turbid yellowish suspension becomes transparent after the addition of $0.45 \mathrm{~mL}$ aqueous $5.0 \mathrm{M} \mathrm{NH} \mathrm{NH}_{4} \mathrm{OH}$ solution and colorless - after the addition of $0.7 \mathrm{~mL}$ aqueous $1.0 \mathrm{M} \mathrm{InCl}_{3}$ solution containing $0.2 \mathrm{M} \mathrm{HNO}_{3}$. Then, $1.0 \mathrm{~mL}$ aqueous $1.0 \mathrm{M}$ $\mathrm{Na}_{2} \mathrm{~S}$ solution was added at stirring and the resulting solution heated in a water bath at $90-95{ }^{\circ} \mathrm{C}$ for $30 \mathrm{~min}$. The CAIS QDS were covered with a $\mathrm{ZnS}$ shell via the decomposition of $\mathrm{Zn}^{\mathrm{II}}-\mathrm{MA}$ complex. For this, $2.0 \mathrm{~mL}$ aqueous 1.0 M MAA solution, $2.5 \mathrm{~mL}$ aqueous $1.0 \mathrm{M} \mathrm{Zn}\left(\mathrm{CH}_{3} \mathrm{COO}\right)_{2}$ solution (with $0.01 \mathrm{M} \mathrm{HNO}_{3}$ ), and $0.3 \mathrm{~mL}$ aqueous $5 \mathrm{M} \mathrm{NH}_{4} \mathrm{OH}$ solution were added at intense stirring to $100 \mathrm{~mL}$ of the core QD solution and the mixture was additionally heated for $30 \mathrm{~min}$. 
The as-prepared CAIS/ZnS QD colloids were concentrated by a factor of $\sim 10$ on a rotary evaporator at around $40{ }^{\circ} \mathrm{C}$ and denoted further as "crude" colloids. In a typical size-selection procedure, to $10 \mathrm{~mL}$ of crude colloids $2.5 \mathrm{~mL}$ of 2-propanol were added to initiate aggregation of the QDs with subsequent centrifugation at $4500 \mathrm{rpm}$ for $5 \mathrm{~min}$. The precipitate was separated and designated further as fraction $\# 1$. This procedure was repeated several times with fresh amounts $(0.5 \mathrm{~mL}$ for each fraction) of 2-propanol producing fractions $\# 2-9$ till the complete exhaustion of QDs in the original crude colloid. Further details of the size-selective precipitation can be found in ref. 27. The collected precipitates \#1-9 were each separately dissolved in $1 \mathrm{~mL}$ of Milli-Q water and stored in the dark at room temperature. For absorption and PL measurements the fractionated solutions were diluted by a factor of $10-500$ (depending on the fraction number) by Milli-Q water.

\section{Characterization}

Absorption and PL spectra were recorded using a UV-vis spectrophotometer Cary 60 and a fluorescence spectrometer Fluoromax 4, respectively, in standard $10.0 \mathrm{~mm}$ optical quartz cuvettes. The PL was excited at $\lambda=420 \mathrm{~nm}$. The PL spectra were normalized to the optical density of the solutions at the excitation wavelength.

The PL QY was determined using AIS/ZnS QDs as a luminescence standard with an absolute QY of $37 \%$ measured using a Fluorolog 3 spectrometer equipped with a Quanta $\Phi$ integrating setup. ${ }^{18}$ The kinetic curves of the PL decays were registered for CAIS/ZnS colloids using a Horiba Jobin Yvon Fluorocube-01-NL. The samples were placed in a $10.0 \mathrm{~mm}$ quartz optical cuvette and excited with a $350 \mathrm{~nm}$ pulse of a NanoLED-350 diode (Horiba Jobin Yvon).

Details on the investigations by X-ray photoelectron spectroscopy (XPS), X-ray diffraction and Raman spectroscopy are provided in (ESI $\dagger$ ).

The photoelectrochemical measurements were performed in a three-electrode cell with $\mathrm{FTO} / \mathrm{TiO}_{2} / \mathrm{QD}$ heterostructures as photoanodes, a Pt counter-electrode, and an $\mathrm{Ag} / \mathrm{AgCl}$ reference electrode. The details of the photoanode preparation and measurements are presented elsewhere. ${ }^{37}$

\section{Results and discussion}

\section{Characterization of CAIS and CAIS/ZnS QDs}

Colloidal CAIS and CAIS/ZnS QDs are characterized by an average hydrodynamic size of $\sim 4 \mathrm{~nm}$ and $\sim 4.2 \mathrm{~nm}$, respectively (ESI, Fig. S1†), with no larger formations present indicating the individual character of each QD in the colloidal ensemble.

The undoped AIS QDs (prepared at $\mathrm{Ag}: \mathrm{In}: \mathrm{S}=1: 7: 10$ ) exhibit a broad PL band centered at $630 \mathrm{~nm}(1.97 \mathrm{eV})$ with a Stokes shift of about $0.4 \mathrm{eV}$ and a spectral width of $\sim 0.35 \mathrm{eV}$ (Fig. 1a and b, curves 1). An increase in the silver content to $\mathrm{Ag}: \mathrm{In}: \mathrm{S}=2: 7: 10$ results in a red shift of the PL band maximum to around $700 \mathrm{~nm}$ (Fig. 2a, curve 1).

As the copper ions are introduced to AIS QDs the PL band shifts to longer wavelengths and gradually loses in intensity
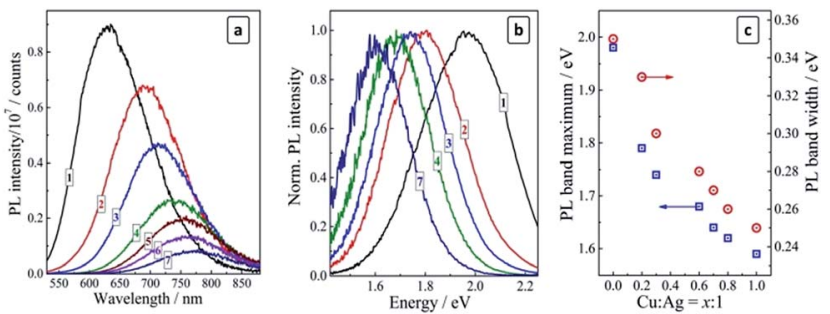

Fig. 1 Original (a) and normalized (b) PL spectra of crude colloids of undoped AIS QDs (curve 1) and CAIS QDs prepared at $\mathrm{Cu}: \mathrm{Ag}=0.2: 1$ (curve 2), $0.3: 1$ (curve 3), 0.6:1 (curve 4), 0.7:1 (curve 5), 0.8:1 (curve 6), and $1: 1$ (curve 7). (c) Maximum position (blue squares) and spectral width (red circles) of the PL band of CAIS/ZnS QDs produced at a different $\mathrm{Cu}: \mathrm{Ag}$ ratio. $\mathrm{Ag}: \operatorname{In}: \mathrm{S}=1: 7: 10$.

(Fig. 1a). Both the red shift and the PL decrease are proportional to the molar $\mathrm{Cu}$ : Ag ratio set at the synthesis. An XPS study (see more details on XPS measurements below) of two selected samples, corresponding to $\mathrm{Cu}: \mathrm{Ag}=1: 10$ and $5: 10$, showed that CAIS QDs purified by the precipitation with 2-propanol and redispersed in DI water, reveal atomic Cu-to-Ag ratios of $(1-2): 10$ and $(4-5): 10$, respectively. Therefore, the copper dopant content in the CAIS QDs is very close to the total amount of copper introduced at the synthesis.

For the ultimate case of $\mathrm{Cu}: \mathrm{Ag}: \mathrm{In}: \mathrm{S}=1: 1: 7: 10$ the PL band maximum can be found at around $780 \mathrm{~nm}(\sim 1.6 \mathrm{eV})$, while the PL intensity is reduced by a factor of about 15 (Fig. 1a, curve 7). The evolution of the PL band from a "yellow" one (centered at around $2.0 \mathrm{eV}$ ) to a "red" one (peaked at $\sim 1.6 \mathrm{eV}$ ) can be visualized by the normalized PL spectra presented in Fig. $1 \mathrm{~b}$ as well as by the dependence of the PL band maximum energy on the copper-to-silver ratio (Fig. 1c, blue squares).

It was reported for the copper-diffused AIS QDs that $\mathrm{Cu}$ can fill the inherent vacancies in the AIS lattice as well to partially substitute silver ions in their lattice sites. ${ }^{30,35}$ The filling of vacancies should be reflected in a narrowing of the distribution of possible electron states participating in the radiative recombination and, hence, in a narrowing of the PL band. Such
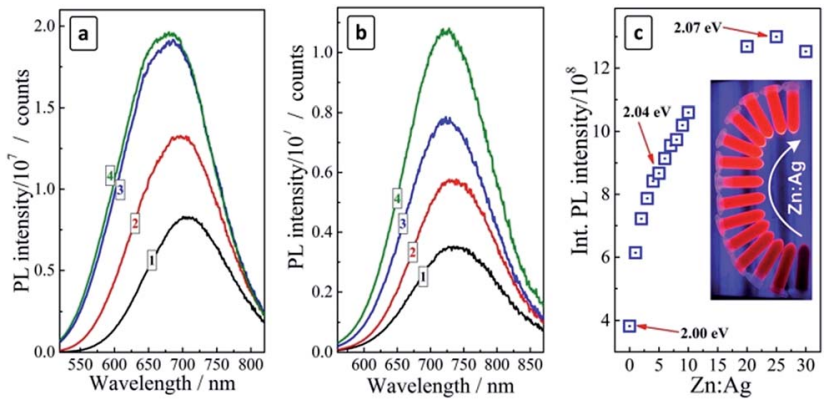

Fig. 2 ( $a$ and b) PL spectra of AIS QDs (a) and CAIS QDs (b) before Zn addition (curves 1) and after the addition at a $\mathrm{Zn}:$ Ag ratio of $2: 1$ (curves 2), $10: 1$ (curves 3), and $20: 1$ (curves 4); (c) integral PL intensity of CAIS/ZnS QDs as a function of $\mathrm{Zn}$ : Ag ratio. Figures on the graph indicate bandgap values for corresponding samples. Insert: photograph of CAIS/ZnS series with an increasing $\mathrm{Zn}$ : Ag ratio under the UV illumination. $\mathrm{Ag}: \operatorname{In}: \mathrm{S}=2: 7: 10$. 
a narrowing is indeed observed in the present case: the spectral width of the PL band decreases from $0.35 \mathrm{eV}$ for the pristine AIS QDs to $0.25 \mathrm{eV}$ for the heavily doped CAIS QDs prepared at $\mathrm{Cu}: \mathrm{Ag}=1: 1$ (Fig. 1c, red circles) indicating that copper does indeed fill the vacancies in the silver-indium-sulfide lattice. A similar narrowing is also observed after the incorporation of $\mathrm{Zn}^{2+}$ ions into the AIS lattice. ${ }^{17,19,23,27,35}$

The deposition of a ZnS shell on the surface of both AIS and CAIS QDs was found to result in a strong PL enhancement (Fig. 2a and b), however, the character of the changes of spectral parameters of the PL band is somewhat different between undoped and doped QDs. As the amount of $\mathrm{ZnS}$ is elevated, the PL band of AIS QDs increases in intensity and shows a "blue shift" (Fig. 2a).

These observations indicate the passivation of the surface defect states participating in non-radiative electron-hole recombination and the diffusion of $\mathrm{Zn}^{2+}$ ions into the lattice of AIS QDs resulting in an increment of the bandgap and the lattice constant..$^{17,18,23,26,27,38}$ In the case of CAIS QDs only a slight blue shift of the PL band is observed along with a PL enhancement (Fig. 2b), supporting the above assumption about a partial filling of the AIS lattice vacancies by copper ions that inhibit the subsequent diffusion of $\mathrm{Zn}^{2+}$. The PL intensity increases by a factor of more than 3 as the $\mathrm{ZnS}$ shell is deposited and the $\mathrm{Zn}$ : $\mathrm{Ag}$ ratio changed from zero to $20-25$ (Fig. 2c). The further increase of the $\mathrm{ZnS}$ content is not accompanied by a further PL intensity increase, rather resulting in a certain decrease of the emission intensity. The highest PL QY obtained for $\mathrm{Zn}: \mathrm{Ag}=25$ was $15 \%$.

\section{Size selection of CAIS QDs}

Recently, we have shown the feasibility of size-selective precipitation of aqueous MA-stabilized AIS and AIS/ZnS QDs by using 2-propanol as a "bad" solvent. ${ }^{27}$ The method is based on the addition of 2-propanol to an aqueous solution of AIS QDS resulting in the precipitation of a fraction of the largest QDS from the colloidal ensemble. The precipitate can be separated and redispersed in pure water forming a colloidal solution stable for months. The repetition of this procedure with an increasing amount of 2-propanol yields many (up to 10-11) fractions of size-selected QDs with distinctly different optical properties and an average size varying from around 2 to 3-3.5 nm. ${ }^{27}$ Here we show that a similar method can be successfully applied for the size selection of the doped MAcapped CAIS and CAIS/ZnS QDs.

Thus, by increasing the volumetric 2-propanol-to-water ratio from 0.3 to 3.3 up to 9 separate fractions of CAIS/ZnS QDs were discriminated from the original colloidal solution $(\mathrm{Cu}: \mathrm{Ag}: \mathrm{In}: \mathrm{S}: \mathrm{Zn}=0.2: 2: 7: 10: 25)$ differing distinctly in their spectral characteristics. The color of the solutions varies from dark-brown for fraction \#1 (the largest QDs) to brightyellow for fraction \#9 of the smallest QDs, while the emission color is changed from deep-red for fraction \#1 to bluish-green for fraction \#9 (see photographs in Fig. 3). As the PL QY and the stability against aggregation of the CAIS/ZnS QDs appeared to be much higher than those for CAIS QDs without protective
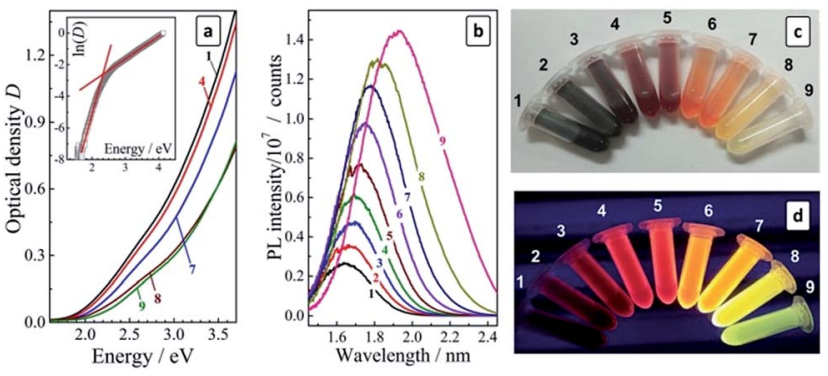

Fig. 3 (a and b) Absorption (a) and PL (b) spectra of size-selected CAIS/ZnS QDs (the curve numbers correspond to the fraction numbers) PL was registered after a normalization of the QD concentration to the same optical density $(\sim 0.1)$ at the $P L$ excitation wavelength. Insert in (a): curve 9 in the coordinates " $\ln (D)$ - quantum energy"; (c and d) photographs of size-selected CAIS/ZnS QD colloids taken under ambient (c) and UV (d) illumination (365 nm).

shells we performed the following studies most exclusively with the core/shell CAIS/ZnS QDs synthesized at the core composition providing the highest $\mathrm{PL}$ QY $(\mathrm{Cu}: \mathrm{Ag}: \mathrm{In}: \mathrm{S}=$ $0.2: 2: 7: 10)$.

A TEM study of size-selected CAIS/ZnS QDs yielded only blurred images (ESI, Fig. S2 $\uparrow$ ), most probably, due to the charging of the sample in the electron beam resulting in oscillations of the sample and compromising the beam focusing. This problem seem to be of a general character for the small aqueous AIS-based QDs observed by us earlier for undoped MA-capped AIS/ZnS ${ }^{27}$ and recently - for the glutathionecapped AIS/ZnS QDs. ${ }^{39}$ Nevertheless, it can be seem from the TEM images that the most populated fractions \#1-2 of the present CAIS/ZnS QDs contain 3-4 $\mathrm{nm}$ particles (in accordance with the DLS measurements), while fractions \#8-9 showed the presence of smaller QDs of $\sim 2 \mathrm{~nm}$ in size.

The absorption spectra of size-selected CAIS/ZnS QDs reveal continuous bands with no distinct maxima or humps and with the band edge shifting continuously to shorter wavelengths as the fraction number increases (Fig. 3a). We have shown earlier ${ }^{27}$ that the absorption band of AIS and AIS/ZnS QDs near the band edge is characterized by a relatively strong contribution of subbandgap states (Urbach absorption) masking the exact position of the band edge. Therefore, a satisfyingly precise determination of the bandgap from the absorption spectra cannot be achieved when the data is replotted in the coordinates of the Tauc equation. In the case of the size-selected series of AIS QDS the contribution of the Urbach absorption increased with decreasing QD size resulting in large uncertainties in the $E_{\mathrm{g}}$ values. We proposed to evaluate the bandgap as the crosspoint of two linear sections of the absorption spectra replotted in the coordinates of the Urbach equation, one of them corresponding to the sub-bandgap absorption and the other - to the interband electron transitions, as shown in Fig. 3a (insert). The values of these bandgaps derived for different fractions using the Urbach equation, $E_{\mathrm{g}}^{\mathrm{U}}$, are collected in Table 1.

The bandgap of the size-selected CAIS/ZnS QDs was found to expand from $2.05 \mathrm{eV}$ to $2.32 \mathrm{eV}$ as the fraction number increased from \#1 to \#9. The energy of the PL band maximum $\left(E_{\mathrm{PL}}\right)$ of the size-selected QDs increases as well, shifting from $1.64 \mathrm{eV}$ to 
Table 1 Some parameters of absorption/PL and metal ratios for a series of size-selected CAIS/ZnS QDs (starting composition Cu : Ag : In : S : Zn $=0.2: 2: 7: 10: 2)$

\begin{tabular}{|c|c|c|c|c|c|c|c|c|c|c|}
\hline Fraction no. & $E_{\mathrm{g}}^{\mathrm{U}}, \mathrm{eV}$ & $E_{\mathrm{PL}}, \mathrm{eV}$ & $\Delta S, \mathrm{eV}$ & FWHM, eV & PL QY, \% & In : Ag & $\mathrm{Zn}: \mathrm{Ag}$ & $\mathrm{C}: \mathrm{Ag}$ & $\mathrm{Cu}: \mathrm{Ag}$ & $\mathrm{Cu}: \mathrm{Zn}$ \\
\hline 1 & 2.05 & 1.64 & 0.41 & 0.31 & 11 & 1.8 & 1.1 & 0.7 & 0.11 & 0.10 \\
\hline 2 & 2.08 & 1.66 & 0.42 & 0.31 & 17 & 1.7 & 1.0 & 0.7 & 0.13 & 0.13 \\
\hline 4 & 2.12 & 1.69 & 0.43 & 0.32 & 28 & 2.2 & 1.0 & 1.0 & 0.08 & 0.08 \\
\hline 5 & 2.13 & 1.71 & 0.42 & 0.35 & 36 & 2.2 & 1.0 & 1.3 & 0.08 & 0.10 \\
\hline 6 & 2.14 & 1.75 & 0.39 & 0.38 & 42 & 3.0 & 1.2 & 1.8 & 0.11 & 0.09 \\
\hline 9 & 2.32 & 1.92 & 0.40 & 0.50 & 58 & 11.0 & 3.0 & 7.5 & 0.27 & 0.09 \\
\hline
\end{tabular}

$1.92 \mathrm{eV}$. Consequently, the Stokes shift, $\Delta S=E_{\mathrm{g}}^{\mathrm{U}}-E_{\mathrm{PL}}$, remains reasonably constant varying slightly around $0.4 \mathrm{eV}$ (Table 1), justifying further the employed method of the bandgap evaluation.

The composition of the size-selected series of the CAIS/ZnS QDs prepared at $\mathrm{Cu}: \mathrm{Ag}: \mathrm{In}: \mathrm{S}: \mathrm{Zn}=0.2: 2: 7: 10: 2$ was analyzed by XPS (second part of Table 1). The atomic $\mathrm{Cu}: \mathrm{Ag}$ ratio was found to vary around 0.1 without sharp deviations matching the original $\mathrm{Cu}: \mathrm{Ag}$ ratio set during the QD synthesis. The $\mathrm{Cu}: \mathrm{Zn}$ ratio was found to be rather stable as well, varying around 0.1 for fractions $\# 1-7$ as expected from the above stoichiometry. These results indicate that the size-selected CAIS/ZnS QDs are characterized by a more or less similar content of the copper dopant throughout the entire series from $\# 1$ to \#7. The last two fractions \#8 and \#9 reveal some deviations from the above tendencies but the amount of QDs in these fractions was very small (by two orders of magnitude smaller than, for example, in fractions \#1 and \#2) which may compromise the accuracy of the XPS measurements.

The ratio of indium to silver was found to increase from around 2 for fraction $\# 1-5$ to $\sim 5$ for fractions \#7, 8 and increasing sharply for fraction $\# 9$ with the smallest CAIS/ZnS QDs (Table 1). In most cases this ratio differs from the preset value indicating that a portion of the $\mathrm{In}^{3+}$ ions is bound to MA and remains unprecipitated in the supernatant solution as well as forms a protective complex ligand layer on the surface of QDs. As shown above, the relative amount of sulfur increases almost linearly with a decrease in the QD size supporting this conclusion. Also, the carbon-to-silver ratio increases showing the same tendency as the In : Ag ratio further supporting the assumption on the partial binding of $\operatorname{In}^{3+}$ ions to the surface complexes with MAA. The $\mathrm{Zn}$ : Ag ratio keeps reasonably stable for fractions \#1-7 indicating that size-selected QDs are characterized by a uniform ZnS shell in different fractions. Some increase in the $\mathrm{Zn}$ : $\mathrm{Ag}$ ratio for the two last fractions can also be an indication that a portion of $\mathrm{Zn}$ (II) binds to MA forming surface protective complexes.

The electron binding energies for In $3 \mathrm{~d}, \mathrm{Ag} 3 \mathrm{~d}$, and $\mathrm{Cu} 2 \mathrm{p}$ derived from XPS spectra are essentially identical for the fractions \#1-9. The In 3d range (not shown here) shows a doublet at $442.2 \mathrm{eV} / 451.7 \mathrm{eV}$ typical for In(III) in ternary copper/silver indium sulfides. ${ }^{40}$ The $\mathrm{Cu} 2 \mathrm{p}$ range (Fig. $4 \mathrm{a}$, insert) exhibits doublet peaks at $931.6 \mathrm{eV} / 951.5 \mathrm{eV}$ for all the studied samples typical for $\mathrm{Cu}(\mathrm{I})$ in CIS compounds. ${ }^{29,40}$ This indicates that the
$\mathrm{Cu}(\mathrm{II})$ ions introduced during the synthesis of the crude colloidal solutions are reduced to $\mathrm{Cu}(\mathrm{I})$ by MAA at the moment of the complex formation as previously observed for aqueous CIS QDs. ${ }^{41}$ A doublet at $367.5 \mathrm{eV} / 373.5 \mathrm{eV}$ in the $\mathrm{Ag}$ 3d range (Fig. $4 \mathrm{~b}$, inset) is typical for $\mathrm{Ag}(\mathrm{I})$ in binary and ternary metal chalcogenides. ${ }^{40}$

The high-resolution XPS spectra of size-selected CAIS QDs in the $\mathrm{S} 2 \mathrm{p}$ range showed a complex band that can be deconvoluted into two (fraction \#2, Fig. 4a) or three (fraction \#7, Fig. 4b) doublets separated by a characteristic spin-orbit splitting of $1.2 \mathrm{eV}$. The first two doublets at $161.2 \mathrm{eV} / 162.4 \mathrm{eV}$ and $163.4 \mathrm{eV} /$ $164.6 \mathrm{eV}$ were assigned to sulfur in the metal-sulfide AIS lattice $\left(S^{2-}\right)$ and to the surface-adsorbed MA species, respectively. ${ }^{40}$

As the fraction number increases (and the QD size gradually diminishes), the intensity of the lattice sulfur signal ( $\left.I_{\mathrm{AIS}}\right)$ becomes weaker while the MA sulfur signal intensity $\left(I_{\mathrm{MA}}\right)$ increases (compare Fig. 4a and b). The intensity ratio $I_{\mathrm{MA}} / I_{\mathrm{AIS}}$ was found to increase in an almost linear way with the increase of the fraction number (Fig. 4c). If a similar surface density of MA ligands is assumed for different QD sizes, this observation may indicate a decrease in the QD size in the consecutive fractions, resulting in an increase in the total surface area of colloidal QDs and in the amount of the surface ligand species as compared to the lattice sulfur atoms. The surface MA concentration depends on the total QD surface area and is proportional to $d^{2}$ ( $d$ is the QD size), while the amount of the lattice sulfur increases as $d^{3}$ and therefore the $I_{\mathrm{MA}} / I_{\mathrm{AIS}}$ ratio should be inversely proportional to the QD size (as $1 / d$ ) and proportional
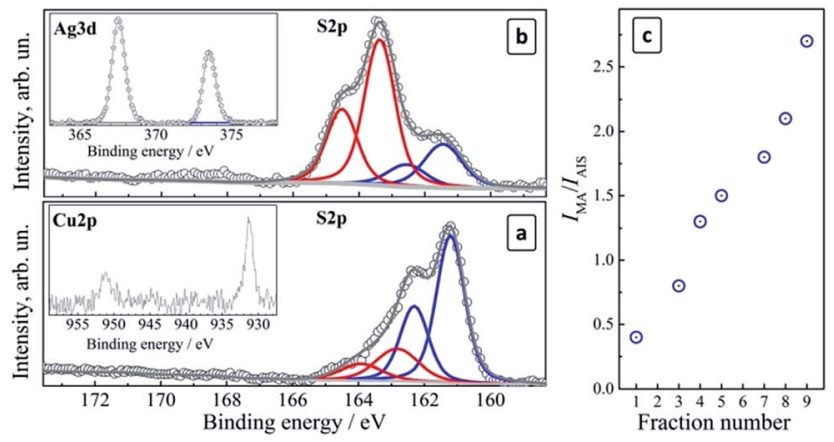

Fig. 4 ( $a$ and b) XPS spectra of CAIS/ZnS QDs in the S $2 p$ range for fractions \#2 (a) and \#7 (b). Inserts: (a) Cu 2p range, (b) Ag 3d range; (c) $I_{\text {MA }} / I_{\text {AIS }}$ ratio as a function of the QD fraction number. 
to the fraction number, in accordance with the observations presented in Fig. 4c.

The X-ray diffractograms of the size-selected CAIS/ZnS QDs reveal diffraction patterns typical for the chalcopyrite silverindium-sulfide compounds for all studied fractions (Fig. 5a). The main (100) peak at $27.5^{\circ}$ is slightly shifted to larger angles (smaller lattice parameters) with respect to pure AIS QDs (26.9 (ref. 27)) indicating that the $\mathrm{Cu}$ doping decreases the lattice parameter of the CAIS QDs because of a smaller ionic radius of $\mathrm{Cu}^{+}$as compared to $\mathrm{Ag}^{+} .{ }^{29}$ The relative intensity of the diffraction peaks decreases somewhat with an increase of the fraction number as a result of an increased disordering in smaller QDs, in accordance with the below-discussed PL observations. The size of the coherent X-ray diffraction domain (close to the average QD size) was estimated by the Scherrer equation to be $2.8 \mathrm{~nm}$ (fraction \#1), $2.0 \mathrm{~nm}$ (fraction \#5), and $1.6 \mathrm{~nm}$ (fraction \#9). This range is very similar to the size range of the non-doped size-selected MA-capped AIS QDs studied by us earlier. ${ }^{27}$ The similarity indicates that the size-selective precipitation produced a more or less identical series of the size-selected QD fractions in both cases provided for the same 2-propanol/water ratio.

A study of the complete set of the size-selected fractions \#1-9 of CAIS/ZnS with Raman spectroscopy was obstructed by the ever-increasing PL background resulting in meaningful spectral features only for the fractions \#1-3. The spectra were found to be identical for all three fractions under two different excitation wavelengths ( 488 and $515 \mathrm{~nm}$ ) and revealed a broadened feature in the range of $240-380 \mathrm{~cm}^{-1}$ with an overtone above $500 \mathrm{~cm}^{-1}$ (Fig. 5b). The main feature can be deconvoluted into two peaks at around $303 \mathrm{~cm}^{-1}$ and $343 \mathrm{~cm}^{-1}$, which is characteristic for AIS compounds..$^{29}$ No other admixture phases were detected in the Raman spectra.

Similarly to the parental colloidal solutions, the size-selected CAIS/ZnS QDs revealed broad PL bands with the intensity increasing strongly with a decrease of the QD size (Table 1). The PL QY was found to increase in a roughly linear manner from $11 \%$ for fraction $\# 1$ up to $58 \%$ for fraction $\# 9$ of the smallest CAIS/ZnS QDs. Fig. 3c and d show that the size selection yields a set of quite intensely emitting luminophors with the emission color varying from deep red for the starting fractions to light-
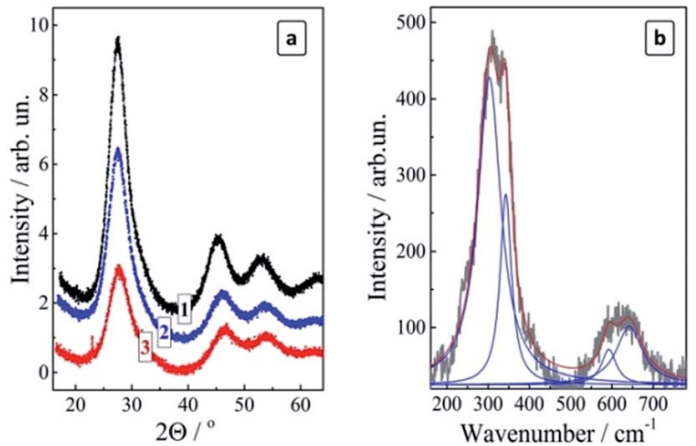

Fig. 5 X-ray diffractograms (a) and Raman spectrum (b) of the sizeselected CAIS/ZnS QDs. Cu: Ag:In: $\mathrm{S}: \mathrm{Zn}=0.2: 2: 7: 10: 2$, (a) fraction \#1 (curve 1), \#5 (curve 2), and \#9 (curve 3), (b) fraction \#3. Blue and red lines in (b) - results of fitting with Lorentz profiles. yellow and yellow-green for the least populated but the brightest fractions \#8 and \#9.

Typically, PL QYs between $\sim 20 \%$ and $\sim 40 \%$ are reported for luminescent AIS/ZnS QDs with the inner AIS core doped with $\mathrm{Zn}^{2+}$ ions. ${ }^{17,19,23,26,38} \mathrm{~A}$ careful optimization of the Ag-to-In ratio in AIS QDs and the AIS-to-ZnS ratio in alloyed ZAIS QDs allowed to reach PL QYs of $60-80 \% .^{18,20,25}$ One of the highest PL QY of $87 \%$ was recently observed for "double-shell" ZAIS/ $\mathrm{ZnIn}_{2} \mathrm{~S}_{4} / \mathrm{ZnS}$ heterostructured QDs. ${ }^{21}$ These figures show that the luminescent size-selected CAIS/ZnS QDs discussed in the present paper are quite competitive with the best reported AIS-based nanoluminophors and, most probably, reveal the highest PL QY reported to date for the copper-doped AIS QDs.

The spectral width of the PL band was found to increase considerably with a decrease in the size of CAIS/ZnS QDs, from $0.31 \mathrm{eV}$ for fraction \#1 up to $0.50 \mathrm{eV}$ for the fraction \#9 of the smallest QDs (Table 1). The same tendency was earlier observed by us for the size-selected undoped AIS/ZnS QDs $^{27}$ and accounted for by an increase disordering of the QDs of decreasing size and a broadening of the spectrum of electron and hole states participating in the radiative recombination. This explanation is corroborated by an increase of the characteristic Urbach energy deduced from the sub-bandgap "tails" of the corresponding absorption spectra. ${ }^{27}$

The dynamics of the radiative recombination in the sizeselected CAIS/ZnS QDs was studied by time-resolved PL spectroscopy for fractions \#1-7 which have almost the same composition as shown by the XPS measurements. The kinetic curves of the PL decays of CAIS/ZnS QDs revealed a distinctly non-monoexponential character typical for ternary QDs. For the fractions of AIS/ZnS and CAIS/ZnS QDs produced at the same volumetric ratio of 2-propanol to water the PL was found to decay faster for the doped QDs as compared with the undoped ones. For example, the radiative lifetime $\tau$ of the QDs was estimated as the time when the original PL intensity decreases by a factor of $e$. It was found to decrease from $405 \mathrm{~ns}$ for AIS/ZnS (fraction \#3) to $355 \mathrm{~ns}$ for CAIS/ZnS QDs (fraction \#3).

This observation along with the reduction in the PL QY induced by copper doping indicates a higher rate of the nonradiative recombination in the doped CAIS/ZnS QDs as compared with AIS/ZnS. An opposite trend was earlier reported for surface-doped AIS/ZnS QDs, ${ }^{35}$ where the PL lifetime increased as a result of $\mathrm{Cu}$ doping. This report along with the present results indicate a crucial role of copper localization, when the relative probability of the $\mathrm{Cu}$ ions participation in radiative and non-radiative recombination depends on the mode of copper introduction producing opposite effects for surface-implanted $\mathrm{Cu}$ ions as in ref. 35 and for $\mathrm{Cu}$ ions introduced on the stage of QD nucleation, as in the present work.

As shown above by the stationary PL measurements the negative influence of $\mathrm{Cu}$ doping on the PL intensity and rate becomes counterbalanced by a decrease in the average size of CAIS/ZnS QDs, resulting in a strong enhancement of the radiative-recombination. The analysis of the stationary PL spectra of the size-selected QDs revealed a steady broadening of the PL band with a size decrease indicative of a broader spectrum of states participating in the PL emission. Therefore, it can 
be expected that the emission becomes faster, which is corroborated by the experimental data indicating a reduction in the radiative life time from $355 \mathrm{~ns}$ to $295 \mathrm{~ns}$ as the fraction number increases from 3 to 7 (Fig. 6a).

By combining data on the stationary PL QY and the average PL lifetimes the rate constants of both radiative and nonradiative recombination in the size-selected CAIS/ZnS QDs, $k_{\mathrm{r}}$ and $k_{\mathrm{nr}}$, can be derived by well-reported approaches ${ }^{31}$ as functions of the QD size (or fraction number). The $k_{\mathrm{r}}$ value was found to grow steadily with a QD size decrease, increasing from $0.63 \times 10^{6}$ to $1.44 \times 10^{6} \mathrm{~s}^{-1}$ as the fraction number increases from 3 to 9 (Fig. 6b). At the same time, $k_{\mathrm{nr}}$ was found to be relatively constant, varying between $2.00 \times 10^{6} \mathrm{~s}^{-1}$ for fraction $\# 3$ and $1.63 \times 10^{6} \mathrm{~s}^{-1}$ for fraction $\# 9$, that is by less than $20 \%$ of the average value, which is too small to assess it as a distinct tendency for a decrease. Therefore, the non-radiative recombination rate constant can be accepted as unchanged in the studied QD size range. The increase of $k_{\mathrm{r}}$ observed for the size-selected CAIS/ZnS QDs with roughly the same composition, $\mathrm{Cu}$ dopant content, $\mathrm{ZnS}$ shell thickness and defect density (the latter derived from unchanged $k_{\mathrm{nr}}$ ) may, therefore, be ascribed to the effect of increased charge carrier confinement favoring the radiative electron-hole recombination. As suggested by the above-discussed XPS data, the amount of In and Zn complexes with MA adsorbed on the QD surface increases with a QD size decrease and the more dense ligand shell can also have some contribution into the observed enhancement of the PL efficiency with the increasing fraction number.

In our earlier studies we have found that the PL efficiency of CIS/ZnS QDs correlates with the photoelectrochemical activity of $\mathrm{TiO}_{2} / \mathrm{CIS}$ nanoheterostructures produced from the same core CIS QDs but without the passivating ZnS shell (or with a very thin one). ${ }^{37}$ In the present work we have investigated the photoelectrochemical activity of $\mathrm{TiO}_{2} / \mathrm{QD}$ composites based on the size-selected CAIS/ZnS QDs introduced as photoanodes in three-electrode cells with a Pt counter-electrode and aqueous $\mathrm{Na}_{2} \mathrm{~S} / \mathrm{Na}_{2} \mathrm{~S}_{x}$ electrolyte, similar to ref. 37 .

The concentration of all the fractions of CAIS/ZnS colloids used for the preparation of the photoanodes was normalized to an equal optical density at $350 \mathrm{~nm}$, that is, far from the
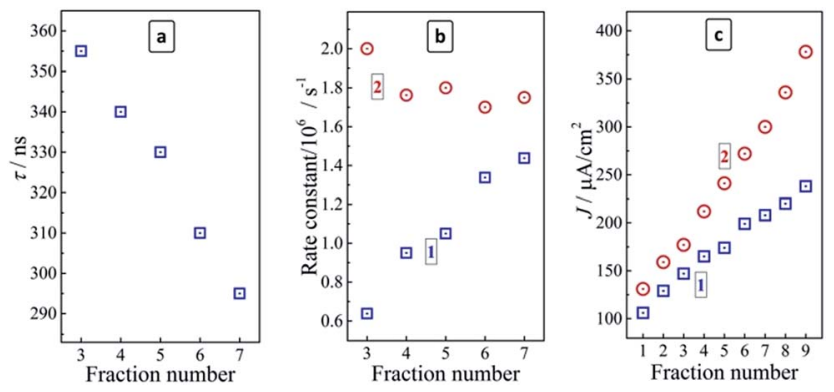

Fig. 6 ( $a$ and b) Average PL lifetime (a), and rate constants (b) of radiative (blue squares 1 ) and non-radiative recombination (red circles 2) a series of the size-selected colloidal CAIS/ZnS QDs. (c) Photocurrent density in the three-electrode photoelectrochemical cells based on $\mathrm{FTO} / \mathrm{TiO}_{2} / \mathrm{CAIS}$ photoanodes with the size-selected AIS/ZnS (blue squares 1) and CAIS/ZnS QDs (red circles 2). absorption band edge, where the absorbance can be assumed to be proportional to the QD concentration and a possible sizedependence of the molar extinction coefficient ignored as a first approximation. In this way, the $\mathrm{TiO}_{2} / \mathrm{QD}$ photoanodes were characterized by roughly the same QD volume density.

It was found that the copper doping enhances the photoelectrochemical activity of AIS QDs since the $\mathrm{TiO}_{2} / \mathrm{CAIS} / \mathrm{ZnS}$ photoanodes produce higher photocurrent densities for all the studied fractions as compared to the undoped $\mathrm{TiO}_{2} / \mathrm{AIS} / \mathrm{ZnS}$ analogues (Fig. 6c). The doping-induced photoactivity increment reveals a dependence on the QD size increasing from $24 \%$ for the largest QDs (fraction \#1) to $\sim 60 \%$ for the smallest CAIS/ZnS QDs (fraction \#9). The photocurrent density produced by $\mathrm{TiO}_{2} / \mathrm{CAIS} / \mathrm{ZnS}$ heterostructures correlates well with the PL QY of the individual colloidal CAIS/ZnS (Table 1) indicating that the PL measurements can be used as a diagnostic tool to assess and predict the light-harvesting efficiency of the QDs introduced into the heterostructured photoanodes of the liquidjunction solar cells.

\section{Conclusions}

The copper-doping of aqueous non-stoichiometric AIS QDs was found to result in a red shift of the PL band maximum from around $630 \mathrm{~nm}$ to $\sim 780 \mathrm{~nm}$ and a PL quenching. The deposition of a ZnS shell onto doped CAIS QDs allows to recover the relatively intense emission with a highest PL QY of 15\% (at a molar $\mathrm{Cu}: \mathrm{Ag}: \mathrm{In}: \mathrm{S}: \mathrm{Zn}$ ratio of $0.2: 2: 7: 10: 25)$ while almost not changing the position of the PL band. The stability of the PL band position in the presence of the protective $\mathrm{ZnS}$ shells in the case of CAIS QDs was attributed to the filling of inherent vacancies in the AIS lattice by $\mathrm{Cu}(\mathrm{I})$ ions impeding the subsequent inclusion of $\mathrm{Zn}$ (II) ions from the shell.

Size-selective precipitation using 2-propanol as a non-solvent allows to discriminate up to 9 fractions of CAIS/ZnS QDs from the original ensemble revealing distinctly different optical properties. The average size of QDs in the fractions changes from about 3 to $2 \mathrm{~nm}$ and smaller similar to the earlier reported case of undoped AIS and AIS/ZnS QDs. ${ }^{27}$ Studies of the fractionated colloids by X-ray photoelectron and Raman spectroscopy showed that the fractions contain only the AIS-like chalcopyrite phase without other phase admixtures.

The decrease of the average size of the CAIS/ZnS QDs results in a blue shift of the PL maximum yielding a series of relatively brightly emitting luminophors with the emission color varying from deep-red to bluish-green and the PL QY increasing from $11 \%$ for the first fraction up to $58 \%$ to the smallest CAIS/ZnS QDs in the least populated fraction \#9.

The rate constant of radiative electron-hole recombination $k_{\mathrm{r}}$ showed a steady increase from $0.63 \times 10^{6}$ to $1.44 \times 10^{6} \mathrm{~s}^{-1}$ with the QD size decrease while the value of the non-radiative recombination rate varied only slightly with the QD fraction number increasing from 3 to 7 . The enhancement of the radiative electron-hole recombination was attributed to the sizedependent spatial exciton confinement in the CAIS/ZnS QDs.

The copper doping was found to result in an enhancement of the photoelectrochemical activity of CAIS/ZnS QDs introduced 
as spectral sensitizers of mesoporous titania photoanodes of liquid-junction solar cells. The doping-induced photoactivity increment increases from $24 \%$ for the largest QDs to $\sim 60 \%$ for the smallest QDs, the photocurrent density correlating closely with the PL QY of original colloidal CAIS/ZnS QDs.

\section{Conflicts of interest}

There are no conflicts to declare.

\section{Acknowledgements}

This work was funded by the European Union's Horizon 2020 research and innovation program under the Marie SkłodowskaCurie grant agreement No 701254 and by The Volkswagen Foundation (project "New functionalities of semiconductor nanocrystals by controllable coupling to molecules"). A. S. N. and I. Y. G. acknowledge financial support from the Russian Ministry of Science and Education (project \# 4.1063.2017/4.6). We acknowledge support by the German Research Foundation and the Open Access Publication Funds of the SLUB/TU Dresden.

\section{Notes and references}

1 A. L. Rogach, A. Eychmüller, S. G. Hickey and S. V. Kershaw, Small, 2007, 3, 536.

2 A. L. Rogach, N. Gaponik, J. M. Lupton, C. Bertoni, D. E. Gallardo, S. Dunn, N. Li Pira, M. Paderi, P. Repetto, S. G. Romanov, C. O'Dwyer, C. M. Sotomayor Torres and A. Eychmüller, Angew. Chem., Int. Ed., 2008, 47, 6538.

3 V. Lesnyak, N. Gaponik and A. Eychmüller, Chem. Soc. Rev., 2013, 42, 2905.

4 L. Jing, S. V. Kershaw, Y. Li, X. Huang, Y. Li, A. L. Rogach and M. Gao, Chem. Rev., 2016, 116, 10623.

5 G. Zu, S. Zeng, B. Zhang, M. T. Swihart, K. T. Yong and P. N. Prasad, Chem. Rev., 2016, 116, 12234.

6 N. Gaponik, S. G. Hickey, D. Dorfs, A. L. Rogach and A. Eychmüller, Small, 2010, 6, 1364.

7 S. R. Thomas, C. W. Chen, M. Date, Y. C. Wang, H. W. Tsai, Z. M. Wang and Y. L. Chueh, RSC Adv., 2016, 6, 60643.

8 H. Azimi, Y. Hou and C. J. Brabec, Energy Environ. Sci., 2014, 7, 1829.

9 F. J. Fan, L. Wu and S. H. Yu, Energy Environ. Sci., 2014, 7, 190. 10 M. Sandroni, K. D. Wegner, D. Aldakov and P. Reiss, ACS Energy Lett., 2017, 2, 1076.

11 M. D. Regulacio and M. Y. Han, Acc. Chem. Res., 2016, 49, 511. 12 S. V. Kershaw, A. S. Susha and A. L. Rogach, Chem. Soc. Rev., 2013, 42, 3033.

13 J. Kolny-Olesiak and H. Weller, ACS Appl. Mater. Interfaces, 2013, 5, 12221.

14 K. E. Knowles, K. H. Hartstein, T. B. Kilburn, A. Marchioro, H. D. Nelson, P. J. Whitham and D. R. Gamelin, Chem. Rev., 2016, 116, 10820.

15 C. Coughlan, M. Ibáñez, O. Dobrozhan, A. Singh, A. Cabot and K. M. Ryan, Chem. Rev., 2017, 117, 5865.
16 P. Reiss, M. Carrière, C. Lincheneau, L. Vaure and S. Tamang, Chem. Rev., 2016, 116, 10731.

17 X. Tang, W. B. A. Ho and J. M. Xue, J. Phys. Chem. C, 2012, 116, 9769.

18 T. Kameyama, T. Takahashi, T. Machida, Y. Kamiya, T. Yamamoto, S. Kuwabata and T. Torimoto, J. Phys. Chem. C, 2015, 119, 24740.

19 G. Gabka, P. Bujak, K. Giedyk, A. Ostrowski, K. Malinowska, J. Herbich, B. Golec, I. Wielgus and A. Pron, Inorg. Chem., 2014, 53, 5002.

20 W. Xiang, C. Xie, J. Wang, J. Zhong, X. Liang, H. Yang, L. Luo and Z. Chen, J. Alloys Compd., 2014, 588, 114.

21 M. Ko, H. C. Yoon, H. Yoo, J. H. Oh, H. Yang and Y. R. Do, Adv. Funct. Mater., 2017, 27, 1602638.

22 T. Torimoto, T. Kameyama and S. Kuwabata, J. Phys. Chem. Lett., 2014, 5, 336.

23 D. Deng, J. Cao, L. Qu, S. Achilefu and Y. Gu, Phys. Chem. Chem. Phys., 2013, 15, 5078.

24 W. W. Xiong, G. H. Yang, X. C. Wu and J. J. Zhu, J. Mater. Chem. B, 2013, 1, 4150.

25 L. Wang, X. Kang and D. Pan, Phys. Chem. Chem. Phys., 2016, 18, 31634.

26 J. Song, C. Ma, W. Zhang, X. Li, W. Zhang, R. Wu, X. Cheng, A. Ali, M. Yang, L. Zhu, R. Xia and X. Xu, ACS Appl. Mater. Interfaces, 2016, 8, 24826.

27 A. Raevskaya, V. Lesnyak, D. Haubold, V. Dzhagan, O. Stroyuk, N. Gaponik, D. R. T. Zahn and A. Eychmüller, J. Phys. Chem. C, 2017, 121, 9032.

28 N. Pradhan, S. D. Adhikari, A. Nag and D. D. Sarma, Angew. Chem., Int. Ed., 2017, 56, 7038.

29 U. Dasgupta, S. K. Saha and A. J. Pal, Sol. Energy Mater. Sol. Cells, 2014, 124, 79.

30 A. Guchhait and A. J. Pal, ACS Appl. Mater. Interfaces, 2013, 5, 4181.

31 S. K. Saha, A. Guchhait and A. J. Pal, Phys. Chem. Chem. Phys., 2014, 16, 4193.

32 F. Li, G. Chen, J. Luo, Q. Huang, Y. Luo, Q. Meng and D. Li, Catal. Sci. Technol., 2013, 3, 1993.

33 S. Shen, L. Zhao, Z. Zhou and L. Guo, J. Phys. Chem. C, 2008, 112, 16148.

34 J. Ke, X. Li, Q. Zhao, Y. Shi and G. Chen, Nanoscale, 2014, 6, 3403. 35 S. Chen, V. Demillo, M. Lu and X. Zhu, RSC Adv., 2016, 6, 51161. 36 A. E. Raevskaya, M. V. Ivanchenko, O. L. Stroyuk, S. Y. Kuchmiy and V. F. Plyusnin, J. Nanopart. Res., 2015, 17, 135.

37 A. Raevskaya, O. Rosovik, A. Kozytskiy, O. Stroyuk, V. Dzhagan and D. R. T. Zahn, RSC Adv., 2016, 6, 100145.

38 T. Torimoto, T. Adachi, K. Okazaki, M. Sakuraoka, T. Shibayama, B. Ohtani, A. Kudo and S. Kuwabata, J. Am. Chem. Soc., 2007, 129, 12388.

39 O. Stroyuk, A. Raevskaya, F. Spranger, O. Selyshchev, V. Dzhagan, S. Schulze, D. R. T. Zahn and A. Eychmüller, J. Phys. Chem. C, 2018, DOI: 10.1021/acs.jpcc.8b00106.

40 A. V. Naumkin, A. Kraut-Vass and S. W. Gaarenstroom, NIST $X$-ray Photoelectron Spectroscopy Database, NIST, Stand. Ref. Database 20, Version 4.1, 2012.

41 Y. Hamanaka, K. Ozawa and T. Kuzuya, J. Phys. Chem. C, 2014, 118, 14562. 